\title{
Thermal Behavior of Critical Micelle Concentration from the Standpoint of Flory-Huggins Model
}

\author{
Kyung-Hee Lim \\ School of Chemical Engineering and Materials Science. Chung-ing Lmiversitw, Seoul 156-756. Korea \\ E-mail: thlimacan ac.kr \\ Received Mav 12, 2009, Accepted Jhly 17, 2009
}

\begin{abstract}
Temperature dependence of the critical micelle concentration ( $\mathrm{CMC}), x_{\mathrm{ClC}}$, in micellization can be described by $\ln x_{C 1 C}=A+B T+C \ln T+D / T$, which has been derived statistical-mechanically. Here $A, B, C$, and $D$ are fitting parameters. The equation fits the $\mathrm{CMC}$ data better than conventionally used polynomial equations of temperature. Moreover, it yields the unique (exponent) value of 2 when the $\mathrm{CMC}$ is expressed in a power-law form. This finding is quite significant, because it may point to the universality of the thermal behavior of $\mathrm{CMC}$. Hence, in this article, the nature of the equation $\ln x_{C}=A+B T+C \ln T+D / T$ is examined from a lattice-theory point of view through the Flory-Huggins model. It is found that a linear behavior of heat capacity change of micellization is responsible for the $\mathrm{CMC}$ equation of temperature.
\end{abstract}

Key Words: Critical micelle concentration. Thermal behavior. Flory-Huggins model. Lattice theory. Heat capacity change of micellization

\section{Introduction}

The capacity to aggregate in solutions is one of the characteristics of surfactants. Micelles are one type of such aggregations and exist above the narrow concentration range called the critical micelle concentration (CMC) where various physical properties of the surfactant solutions change abruptly.

Micellization is affected by various factors such as the physical properties of surfactant species (hydrophobic volume. chain length, head group area). temperature, pressure. ionic strength. $\mathrm{pH}$. etc. Among them temperature has been one of the focal points. since changes in CMC with temperature can provide information on molecular interactions. Accordingly. numerous studies on CMC versus temperature have been done so far. ${ }^{3}$ For nonionic surfactants the CMC decreases with temperature due to an increase in hydrophobicity caused by the destruction of hydrogen bonds between water molecules and hydrophilic groups." Therefore the $\log \mathrm{CMC}$ of nonionic surfactants $v s .1 / T$ plot is nearly linear.

However, for ionic surfactants the CMC display's a U-shaped behavior with temperature. ${ }^{3.4}$ The CMC minimum is characterized by the mole fraction. $\dot{x}_{\text {ar }}$ of the surfactant and the temperature. $T^{*}$ at $x^{*}$ CIC. Furthermore. the effect of temperature on $\mathrm{CMC}$ can be represented by a power law expression

$$
\left|x_{C I T C}-x_{C I C}^{*}\right|=\mathrm{A}_{\text {power }}\left|T-T^{*}\right| n \text {. }
$$

It has been a common practice to analyze the measured CMC data $x_{C I C}(T)$, with temperature using poly nomial equations of the type

$$
\ln x_{C M C}=a+b T+c T^{2}+d T^{3}+d T^{4}+d T^{5}+d T^{6}+\cdots .
$$

However Lim et al. have found that. when Eq. (2) is used for the power-law expression of Eq. (1), the values of the exponent $n$ changes substantially with the number of terms used even for a fixed surfactant system. ${ }^{5}$ For example, when the $4^{\text {th }}-.5^{\text {th }} \cdot$. and $6^{\text {th }}$-order poly nomials are used in Eq. (2) for the cationic surfactant octadecyl trimethyl ammonium chloride (OTAC). molar concentration $\bar{c}_{\mathrm{CHC}}^{-}$at the CMC numimun, the temperature $T^{*}$, and the exponent $n$ are obtained as ${ }^{6 *}$

$$
\begin{aligned}
& 0.327 \mathrm{mM}, 23.6^{\circ} \mathrm{C}, 1.48: 0.322 \mathrm{mM}, 23.6^{\circ} \mathrm{C} .1 .56 \text { : } \\
& 0.323 \mathrm{mM}, 24.7^{\circ} \mathrm{C}, 1.12 \text {. }
\end{aligned}
$$

These results show that, when $c_{c u c}^{*}$ 's differ only by $1.6 \%$ $(0.327 \mathrm{mM}$ us $0.322 \mathrm{mM})$, the exponents $n$ 's differ by significant $5.4 \%$ (1.48 $\mathrm{ks} .1 .56$ ). Moreover, when $T^{*}$ s differ by $4.6 \%(23.6 \mathrm{v}, 24.7) . n$ 's differ by substantial $33 \%(1.56 \mathrm{vs}$. 1.12). Hence. if poly nomial equations of Eq. (2) are used for the power-law expression, inconsistent $n$ values are obtained for a given surfactant. These results strongly suggest that the polynomial equations of temperature be inadequate for the power-law expression and that other suitable equations be needed for this matter.

Some developments have been made in this direction. Muller introduced the following equation: ${ }^{8}$

$$
\ln x_{\circlearrowleft \mathrm{C}}=A+B T+C \ln T \text {. }
$$

Here A. B. and $C$ are coefficients or fitting parameters. Meanw hile Lim et al proposed

$$
\ln x_{\triangle \mathrm{C}}=A+B T+D / T \text {. }
$$

which was derived employing thermody nanuc equations on micellization and some observed ty pical phenomena in micellization. such as contpensation phenomena and linear dependence of enthalpy of nucellization on temperature. ${ }^{5,9}$ and also proposed 


$$
\ln x_{C M C}=A+B T+C \ln T+D / T,
$$

which was derived from statistical-mechanical analỵsis of micellization. ${ }^{[1.14}$

Compared to the polynomial equation. Eq. (2). of temperature. Eqs. (4) to (6) yield better fitting results for a given surfactant sy stem: the correlation coefficient of the latter is much closer to 1 than that of the former. What is much more important is that the last three equations y ield $n=2$. irrespective of surfactant system. ${ }^{1+}$ That is $n=2$ appears to be universal for the (normal) micellization. This finding is quite significant and may shed light on better. theoretical understanding of micellization.

If the exponent $n$ is found with the polynomial equations of temperature. $n$ appears to depend on the surfactant system. For example. La Mesa found that $n=1.73 \pm 0.03$ for nine ionic surfactants. ${ }^{15}$ In contrast. Stasiuk and Schramm ${ }^{16}$ obtained $n=$ 3.54 for three commercial ionic surfactants and $n=5.80$ for two commercial amphoteric surfactants. These results would point that the exponent $n$ is characteristic to the surfactant system. Then, the CMC must be measured at various temperatures for a given surfactant system to examine the effect of temperature on the CMC. However, if $n=2$. irrespective of surfactant system. then pre-exponential factor $A_{\text {pore }}$ in Eq. (1) would reflect the characteristics of the surfactant system under examination. This would enable us to determine CMC at an arbitrary temperature with much fewer experimental data.

$\mathrm{Eq}$. (6) appears that it is obtained by combining Eqs. (4) and (5). In fact. Eq. (6) can be obtained from Eq. (4) or (5) with the assumption that the degree of counterion binding, $\beta$. depends linearly with temperature $\left(\beta=\beta_{0}+\beta_{1} T\right)$. Since Eqs. (4) and (5) are semi-theoretical and $\mathrm{Eq}$. (6) is relatively rigorous. one may take the latter more seriously. It is quite necessary to establish that the basis of Eq. (6) is solid and that $n=2$ is correct. For that matter significance of $\mathrm{Eq}$. (6) is examined from latticetheoretical standpoint in this article.

Cationics are known to exhibit excellent antistatic effects and softness. OTAC is probably the most commonly used in such applications. However. little has been conducted on the micellization of this surfactant. This was the reason OTAC was selected as the surfactant. ${ }^{6}$ and a part of the Lim et al. 's results are used in this article.

\section{Experimental}

Materials. The cationic surfactant octadecyltrimethy lammonium chloride (OTAC) was purchased from Fluka. The surfactant had a stated purity of $98 \%$. It was recrystallized twice from absolute ethanol for further purification. The purified surfactant was finally dried in an evacuated desiccator. The water used was distilled and deionized.

Electrical Conductivity Measurements. The critical micelle concentrations (CMCs) of OTAC were determined from the electrical conductivities of its aqueous solutions. For the conductivity measurements of the surfactant solutions. a Radiometer (Paris, France) Model CDM 210 conductivity meter and a Model CDC6+1T conductivity cell with platinized electrodes were used. The conductivity cell was calibrated with standard $\mathrm{KCl}$ solutions. The surfactant concentrations were changed by the addition of deionized water from a burette to the surfactant solution. which was contained in a jacketed. thermostatted beaker. The surfactant solution was mixed thoroughly using a magnetically driven stirrer. The temperature was controlled within $0.1{ }^{\circ} \mathrm{C}$ by a microprocessor-installed thermostat bath (Model VS-1205WP-CWO. Vision Scientific. Seoul. Korea).

\section{Theory}

Gibbs Free Enelgy of Micellization, $\Delta G_{m i c}{ }^{5}$ Thermodynanics of micellization has been often described by the models of mass action law and phase separation. In this article I take the closed association model. which is popular one of the mass action law models. and which provides the essence of micellization without loss of generality: In this model it is assumed that monodisperse nucelles comprised of $n$ surfactant molecules are found. which are formed wia the following reaction

$$
\begin{aligned}
& n S+m G=\left(S_{n}\left(G_{m}\right)^{=}=M^{z}\right. \\
& \text { surfactaunt comuterous micelle }
\end{aligned}
$$

where $S . G, M$, and $z$ stand for surfactant monomer. counter (gegen) ion. micelle, and the charge or the valence of the nucelle. respectively: The equilibrium constant $K$ for the reaction is

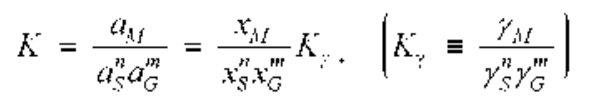

in which $a$, is the activity of the species $i$. Use is made in Eq. (8) of the relation $a_{i}=y_{i} x_{i}$ with $\gamma$ and $x$ being the activity coefficient and mole fraction, respectively. If we assume that $K_{:}=1$ or ideal system. then the activities can be replaced by the mole fractions and therefore $\mathrm{Eq}$. (8) becomes

$$
K=\frac{x_{i t}}{x_{s}^{n} x_{G}^{m}}
$$

For the monomer. $a_{S}=1$ amounts to assuming that the departure from ideal behavior is only the aggregation process. In principle. it can be removed by estimating activity corrections from solution theory. However. it is quite problentatic to assume ideal behavior for the nucelles (i.e., $\gamma_{M}=1$ ) because of the large size difference between monomers and micelles. ${ }^{18}$ The micelles will also interact strongly and for ionics the interaction will become very significant as soon as the mean separation is less than about $8-10$ times double layer thickness that occurs at surfactant concentrations not far above the $\mathrm{CMC}$. It must also be noted that. when ionic micelles are formed. there is a strong tendency for the counter ions to be associated closely with the head groups. because of the high electrical potential in that region. This is another source of nonideality. Notwithstanding the problems attributed to the assumption $\gamma_{i}=1$. Eq. (9) still represents nicellization quite well. ${ }^{19-1}$

The standard Gibbs free energy change, $J G^{\circ}$, for the micellization of $\mathrm{Eq}$. (7) can be obtained from the well-known 
thermody namic result $\Delta G^{\theta}=-R T \ln K$. From these equations along with Eq. (9) one obtains

$$
\Delta G^{\theta}=-R T \ln K=-R T\left(\ln x_{i t}-n \ln x_{s}-m \ln x_{G}\right)
$$

Here $J G^{G}$ is also expressed as

$$
\Delta G^{\theta}=\mu_{M}^{e}-n \mu_{S}^{\theta}-m \mu_{G}^{e}
$$

in which $\mu_{i}^{\dot{\theta}}$ is the standard chemical potential of species $i$. If we define $\Delta G_{\sigma^{\theta} \rightarrow>}$ as the standard Gibbs free energy change of micellization per mole of strfactant. i.e. $\Delta \underline{G}_{m c=n}^{\theta}=\Delta G^{\theta} / n$, then

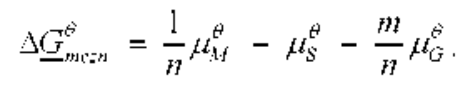

and

$$
\Delta \underline{G}_{m i: n}^{S}=-\frac{R T}{n} \ln K=-R T\left(\frac{1}{n} \ln x_{n}-\ln x_{S}-\frac{m}{n} \ln x_{G}\right)
$$

Introducing the ratio $\sigma=x_{M} / x_{\text {OlC }}$ as Tanford did $^{\text {*2 }}$ and recognizing that, at the $\mathrm{CMC}, x_{S}=x_{C h C}-x_{M}$ and that $x_{G} \approx x_{S}$ ( $=x_{\text {Cac }}$ ) when the ionic surfactant $S_{v_{s}} G_{k=}$ is a symmetric electrolyte. i.e., is $=1_{G}$, we obtain

$$
\frac{\Delta \underline{G}_{m i n}^{G}}{R T}=\left(1+\frac{m}{n}-\frac{1}{n}\right) \ln x_{C H C}-\frac{1}{n} \ln \sigma+\ln (1-\sigma) .
$$

The choice of $\sigma$ has only a small effect on the free energy of micellization because it ranges usually from 0.01 to 0.10 . Moreover, for large $n$ (for example, $n \geq 50$ ) the $1 / n$ terms and the last term in the right-hand side are negligible. Hence, in this case Eq. (I4) is reduced to

$$
\frac{\Delta \underline{G}_{m c=n}^{\theta}}{R T}=(1+\beta) \ln x_{C 1 C}
$$

where $\beta \equiv m / n$ is the degree of counter ion binding. This is the relationship between $\Delta \underline{G}_{m i z / s}^{\theta}$ and $x_{C M C}$ for micellization. Although the degree of counter ion binding. $\beta$. is usually a weak function of temperature it can be taken as constant without loss of generality.

Statistical-Mechanical Derivation of $\ln x_{C \%}(T) .^{13-1+}$ For a micellar solutions consisted of ${ }_{\text {us }}$ solvent (water) molecules. $N_{1}$ surfactant monomers, and $N_{i s}$ micelles with association mumber $s$, the partition function $Q$ may be described by

$$
Q=\frac{q_{w}^{N_{w}}}{N_{w} !} \frac{q_{1}^{N_{1}}}{N_{1} !} \prod_{s=2}^{\infty} \frac{q_{s}^{N_{M s}}}{N_{M s} !}
$$

where $q_{i}$ is the particle partition function of species $i$. After $q$ 's are found accounting for the essential phenomena in micellization (such as hyddrophobicity. electrical interaction. van der Waals interaction, translational. rotational, vibrational motions. electronic contribution. etc), the partition function $Q$ is obtained. The micelle șystem under consideration has obviouly the following constraints:

$$
\begin{aligned}
& N_{s t}=\sum_{s=1}^{\infty} s N_{s / s}=\sum_{s=1}^{\infty} s\left(N_{s} / s\right)=\sum_{s=1}^{\infty} N_{s} \\
& N=N_{w}+X_{1}+\sum_{s=2}^{\infty} X_{M S}
\end{aligned}
$$

Here $N_{s t} N_{k s}, N_{s,}, N_{w}, N_{1}$ and $N$ are, respectively. the total number of surfactant molecules. number of micelles of size $s$, total number of surfactants in the nucelles. number of solvent (water) molecules, number of surfactant monomers, and the total number of particles. Eqs. (17) and (18) are simply expressions of the mass conservation law.

At the equilibrium of the micellization process. the Helmholtz free energy $F$ is minimun. This inplies that the following equation should be satisfied:

$$
0=\frac{\partial F}{\partial N_{s}}=\frac{\partial F}{\partial N_{s}}\left[\ln Q+\dot{\lambda}\left(\sum_{s=1} s N_{s i s}-N_{s t}\right)\right](s=1,2, \ldots, \infty)
$$

where $i$ is a Lagrange multiplier. Since $\lambda_{5}$ can be represented by $N_{1}$ and $N_{3,}$, Eq. (19) can be expressed in terms of $\partial F / \partial V_{1}$

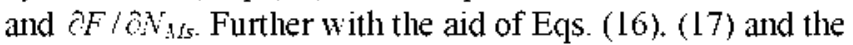
conconditi of $F\left(N_{n}, N_{1}, N_{s, 1}\right)=F\left(N_{n}, N_{1}, N_{s}, s\right)$ at the critical micelle concentration (CMC). ${ }^{23} \mathrm{Eq}$. (19) leads us to

$$
\ln H_{u s}=\ln \frac{q_{s}}{q_{1}^{s}}-s \frac{\partial \ln q_{s}}{\partial s}-s \gamma_{1} \frac{\partial \ln q_{1}}{\partial \gamma_{1}}+s \ln X_{1}
$$

Now for a concret expression of Eq. (20) $q_{1}$ and $q_{s}$ should be elucidated. Accounting for translational. rotational. vibrational motions along with hydrophilic head group interactions, hydrophobic van der Waals interactions, and interactions between the hydrophobic tails and solvent. we have determined $q_{1}$ as follows.

$$
\ln q_{1}=3 \ln n_{\mathrm{CH}_{2}}+3 \ln T+0.3 n_{\mathrm{CH}_{2}}-c_{1}^{u l} \frac{n_{\mathrm{CH}_{2}}}{T}+\ln \Gamma_{f}+c_{\mathrm{l}}
$$

where ${ }^{\mathrm{CH}_{2}}$ is the number of carbons in the hydrophobic chain, $r_{f}$ is the free volume. and $c_{1}^{i l '}$ and $c_{1}$ are constants uniquely given in terns of molecular properties. For $q_{s}$ we have accounted for solvation of head groups. intramicellar electrostatic effects. hydrophobic bonding of tails as well as translational, rotational. vibrational motions and came up with

$$
\begin{aligned}
& \ln q_{s}=3(s+1) \ln n_{\mathrm{CH}_{2}}+(2 s+1) \ln T+\ln I_{f}+c_{\mathrm{exT}} \\
& +3 \ln s+s+c_{3 p h \hat{h}}\left(s-s^{2: 3}\right)-c_{e l s c} \frac{s^{5: 3}}{n_{\mathrm{CH}}{ }^{1: 3} T}+(s-1) \ln c_{\text {int }}
\end{aligned}
$$

Here also, the constants $c_{\text {sxr. }} . c_{\text {hph }} . c_{\text {sle }}$. and $c_{\text {int }}$ are given uniquely for the surfactant șistem. 
The conventional concentration $C$ in the unit of moles per liter is related to the $N_{s}$ and $\sigma_{f}$ by the relation

$$
\frac{N_{s}}{V_{f}}=\frac{C \cdot N_{A v} \cdot 10^{-3}}{f_{v}}
$$

with $N_{A k}$ being the Arogadro's number and $f_{r}$ being the fraction of free volume to the total volume. Hence. putting into Eqs. (21) to (23) into $\mathrm{Eq}$. (20) and solving for $C(=\mathrm{CMC}$ ) yields

$$
\begin{aligned}
& \ln C H C=(1-3 s) \ln n_{C H_{2}}+(1-3 s) \ln T+s \ln T_{f} \\
& +4 \ln s-\frac{c_{l p p h}}{3} s^{2: 3}+\frac{2}{3} c_{e l e t} \frac{s^{5: 3}}{n_{\mathrm{CH}_{2}}{ }^{1 / 3} T}+s \ln N_{1} \\
& +0.3 s n_{\mathrm{CH}_{2}}-c_{1}^{\prime \prime} s \frac{n_{\mathrm{CH}}}{T}-c_{1} s+c_{m c}
\end{aligned}
$$

Taking into consideration $c_{h p b}=a+b T+c / T$ given by Nemethy and Schraga ${ }^{2+}$ along with Eq. (24) we obtain $x_{\mathrm{CMC}}$ as

$$
\ln x_{\mathrm{CMC}}=A+B T+C \ln T+\frac{D}{T}
$$

Although the contants appeared in Eq. (21) through Eq. (24) are all given uniquely, the coefficients $a, b, c$ are not so. Therefore $A, B, C$ and $D$ in Eq. (25) are fitting parameters. which depend on the surfactant systems. Now the combination of Eqs. (15) and (25) yields the following equation:

$$
\frac{\Delta G_{m b=13}^{\phi}}{R T}=(1+\beta)\left(A+B T+C \ln T+\frac{D}{T}\right)
$$

Free Energy of a Polymer Solution by Lattice Theory. A polymer solution comprised of $N$, solvent (water) molecules and $N_{p}$ polymer molecules may be represented by a lattice. Each polymer molecule has 1 segments and therefore the number of total lattice points is $N_{w}+1 N_{p}\left(=N_{L T}\right)$. Since a solvent molecule or a polymer segment occupies one lattice point, the (volume) fractions $\phi_{w}$ of the solvent and $\phi_{p}$ of the polymer are respectively.

$$
\phi_{w:}=\frac{N_{w}}{N_{L T}}=\frac{N_{w}}{N_{w}+1 N_{p}} \text { and } \phi_{p}=\frac{N_{p}}{N_{L T}}=\frac{1 N_{p}}{N_{w}+1 N_{p}}
$$

If a solvent molecule and a polymer has $n_{i}$ nearest neighbors and the interaction energy between particle $j$ and particle $k$ is $\varepsilon_{j k}$, then the energy $E$ of the poly mer solution can be described as

$$
\begin{aligned}
& E=\frac{1}{2} N_{L T} \sum_{j, k=1}^{2} \phi_{J}\left(n_{i} \phi_{k}\right) \varepsilon_{J k} \\
& =\frac{n_{3} N_{L T}}{2}\left(\varepsilon_{11} \phi^{2}+\varepsilon_{12} d \phi_{2}+\varepsilon_{2-1} \hat{z}\right) \text {. }
\end{aligned}
$$

Since $E$ is found the canonical partition function $Q$ of the polymer solution is obtained as

$$
Q=\left[q_{n}(T)\right]^{N_{k}}\left[q_{p}(T)\right]^{* p} \sum_{E} g\left(N_{w}, N_{p}, r\right) e^{-\beta E}
$$

where $g\left(N_{n}, N_{y^{*}} v^{\cdot}\right)$ is the degeneracy at the energy $E$.

The essential point of Flory-Huggins model lies in the way of calculating $g\left(N_{n}, N_{p} .1^{\prime}\right)$. According to the model $g *\left(N_{w}\right.$, $N_{p, 1}$ ) for the maxinum term in the summation of $\mathrm{Eq}$. (29) is given approxinutely by

$$
\begin{aligned}
g^{*}\left(N_{w}, N_{p}, 1^{\prime}\right) & \approx \frac{N_{L T}^{N_{n}+N_{p}}\left[n_{c}\left(n_{c}-1\right)^{r-2}\right]^{N_{p}}}{N_{n} ! N_{p} !} e^{-N_{L T}} \\
& =\frac{N_{L T} !}{N_{w} ! N_{p} !}\left(\frac{n_{c}-1}{N_{L T}}\right)^{(v-1) N_{p}}
\end{aligned}
$$

and therefore one can obtain Helmholtz free energy of the polymer solution enploying the characteristic equation. $F\left(N_{w}, N_{p}, l^{\prime}\right)=-k T \ln Q^{*}$. of the canonical ensenble. Here $Q *$ is the partition function after the summation in Eq. (29) is replaced by the maximum term. Then mixing function $\Delta F_{m i x}$ can be found as

$$
\begin{aligned}
\Delta F_{\text {mix }} & =F\left(N_{w}, N_{p}, 1^{\prime}\right)-F\left(N_{n}, 0,1\right)-F\left(0, N_{p}, 1\right) \\
& =N_{L T} k T\left(\phi_{w} \cdot \ln \phi_{w^{\prime}}+\frac{\phi_{p}}{\mathrm{I}^{\prime}} \ln \phi_{p}+\chi \phi_{w} \phi_{p}\right) .
\end{aligned}
$$

or in one mole basis $\left(N_{L T}=N_{\text {tr }}\right.$ with $N_{\text {tr }}$ being the Avogadro's number)

$$
\frac{\Delta \underline{F}_{m i x}}{R T}\left(=\frac{\Delta \underline{G}_{m i x}}{R T}\right)=\phi_{w} \ln \phi_{n}+\frac{\phi_{p}}{1} \ln \phi_{p^{\prime}}+\chi \phi_{w} \phi_{p^{\prime}},
$$

where $y$ is the interaction parameter, defined by

$$
\begin{aligned}
& \chi=\frac{n_{c}\left(\varepsilon_{n p}-\varepsilon_{w h} / 2-\varepsilon_{p p} / 2\right)}{k T}=\beta n_{c} \omega . \\
& {\left[\omega \equiv \varepsilon_{w p}-\varepsilon_{w\}} / 2-\varepsilon_{p p} / 2\right]}
\end{aligned}
$$

The first equality in $\mathrm{Eq}$. (32) is the reflection of the fact that in the lattice theory Helnholtz free energy and Gibbs free energy are equivalent.

\section{Results and Discussion}

Electrical conductivities of aqueous OTAC solutions at different surfactant concentrations and temperatures are shown in Figure 1. At each temperature the electrical conductivity exhibits an excellent linear relationslip with surfactant concentration. The break point or the point of intersection of the two conductivity lines are taken as the CMC. Figure 2 shows the CMCs thus determined at various temperatures. Ionic surfactants have been known to display a U-shaped behavior in a CMC-temperature plot. The CMCs of the cationic OTAC surfactants also exhibit this behavior. The $\mathrm{CMC}$ decreased to the minimum point and then increased with temperature 


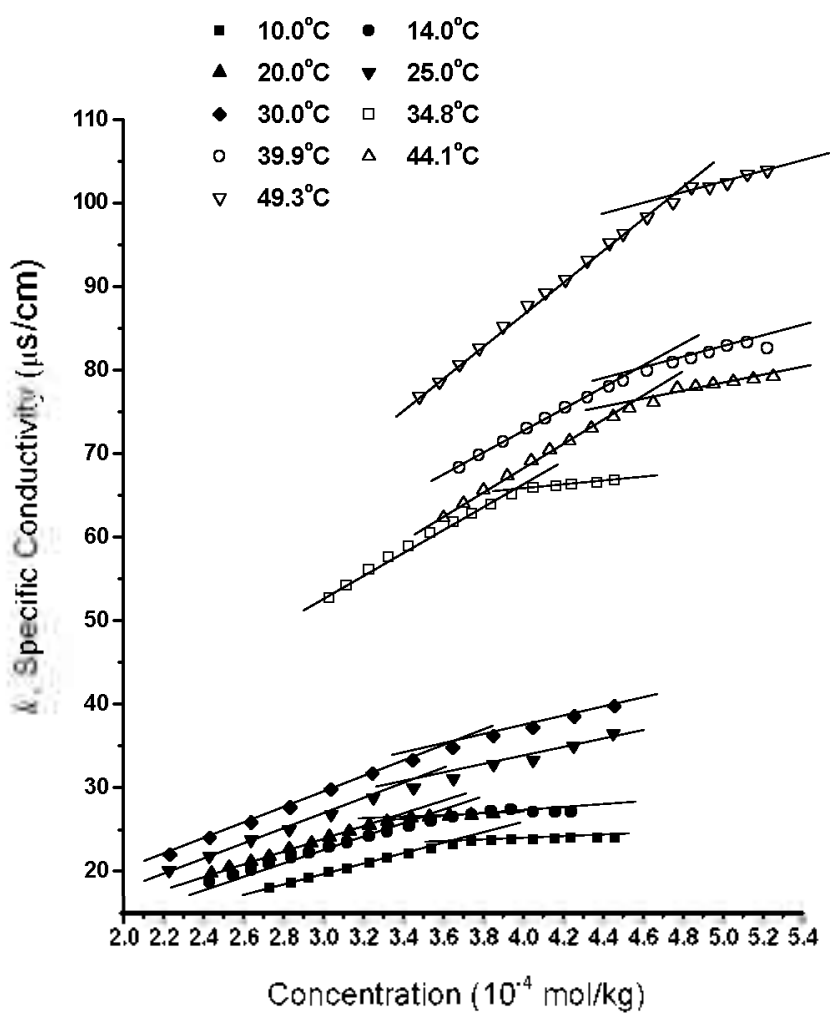

Figure 1. Electrical conductivities of aqueous OTAC solutions at different surfactant concentrations and temperatures.

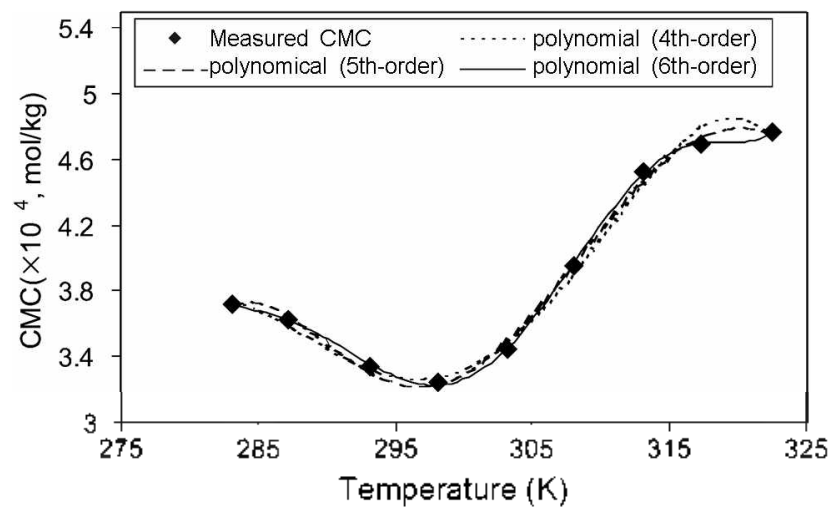

Figure 2. Fits of the $4^{\text {th }}, 5^{\text {th }}$, and $6^{\text {th }}$ polynomial equations to the CMC-temperature data for aqueous solutions of the cationic OTAC.

Also in Figure 2, fits of $4^{\text {th }}-.5^{\text {th }}$-, and $6^{\text {th }}$-order polynomial functions to the $\mathrm{CMC}$ data are shown. The polynomials fit the data excellently, as indicated by the correlation coefficients of 0.99 . However as stated in Introduction. quite different $n$ 's are obtained and $n$ appears to be sensitive to the order of the poly nomial used. Among three values (1.48. 1.56. and 1.12) of $n$. which one we choose is quite arbitrary. However, this is not the case with Eqs. (4). (5), and (6). If these equations are used for Eq. (1), the exponent $n$ is always equal to 2 . When these equations are used for other surfactants, the exponent $n$ is 2 consistently. Thus, the exponent $n$ takes only one value of 2 , irrespective of surfactant. This result is quite significant.

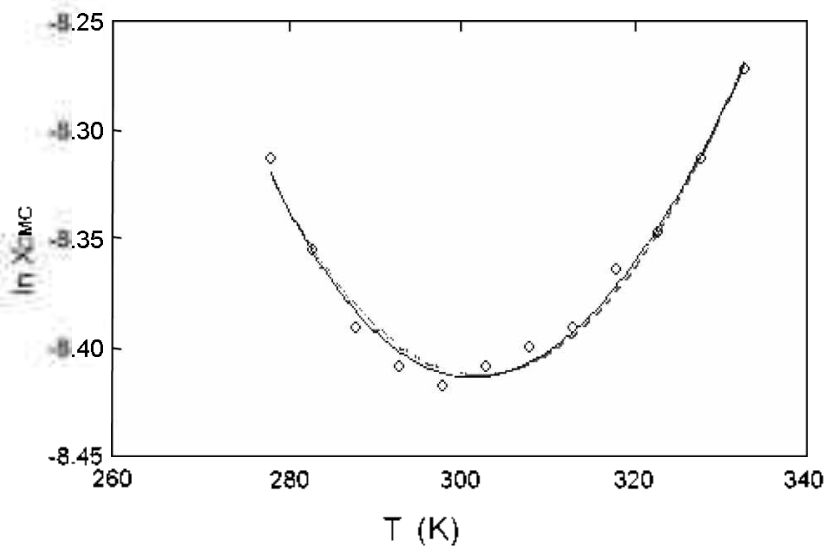

Figure 3. Comparison of tits by $2^{\text {tud }}$-order polynomial equation and by Eq. (4) for dodecyl-4-methoxypyridinium chloride open circle, measured; $;^{-1}$ Dashed line, fit by $2^{\text {ind }}$-order polynomial; Full line, fit by Eq. (4).

because $n=2$ appears to be a universal property for micellar solutions. Moreover. Eqs. (4), (5), and (6) y ield better fitting results. Figure 3 shows the fits of Eq. (4) and the $2^{\text {nst }}$-order polynomial $\ln x_{C \mathrm{C}}=a+b T+c T^{2}$ to the OTAC CMC data. Both equations (with the same number of the fitting coefficients) fit the data well and Eq. (4) fits better than the $2^{\text {tikl }}$-order polynomial, as indicated by the correlation coefficients, $0.9958 \mathrm{rs} .0 .9883$.

The fact that the unique value of $n=2$ is provided by Eq. (6) is quite significant. Hence, the nature of Eq. (6) should be scrutinized. One of the basic thermodynamic relation is $J C_{p}=$ $(\delta \triangle H / \partial T)_{F}=T(\partial \unlhd S / \partial T)_{F}$. It is generally considered that $\Delta$ $C_{P}$ depends linearly with temperature. i.e. $\backslash_{C p}=a_{C_{p}}+b_{C_{p}} T$ for polymer systems. ${ }^{25,26}$ Then, from $\Delta C_{F}$ we obtain $\underline{\perp H}$ and $\triangle S$ from these equations as

$$
\begin{aligned}
& \Delta H=\int C_{F} d T=a_{C p} T+\frac{b_{C p}}{2} T^{2}+\text { const }_{H} \\
& \underline{\Delta S}=\int \frac{C_{P}}{T} d T=a_{C p} \ln T+b_{C p} T+\text { const } t_{S} .
\end{aligned}
$$

Since $\Delta \underline{H}$ and $\Delta S$ are at hand. $\Delta \underline{G}$ can be easily calculated as

$$
\begin{aligned}
\Delta G & =\Delta \underline{H}-T \Delta S \\
& =\text { const }_{H}+\left(a_{C p}-\text { consts }\right) T-a_{C p} T \ln T-\frac{h_{C p}}{2} T^{2}
\end{aligned}
$$

or

$$
\begin{aligned}
\frac{\Delta G}{R T} & =\frac{a_{C p}-\text { const }_{S}}{R}-\frac{b_{C p}}{2 R} T-\frac{a_{C p}}{R} \ln T+\frac{\text { const }_{H}}{R} \frac{1}{T} \\
& =A+B T+C \ln T+\frac{D}{T}
\end{aligned}
$$

where the coefficients $A, B, C$ and $D$ are defined as 


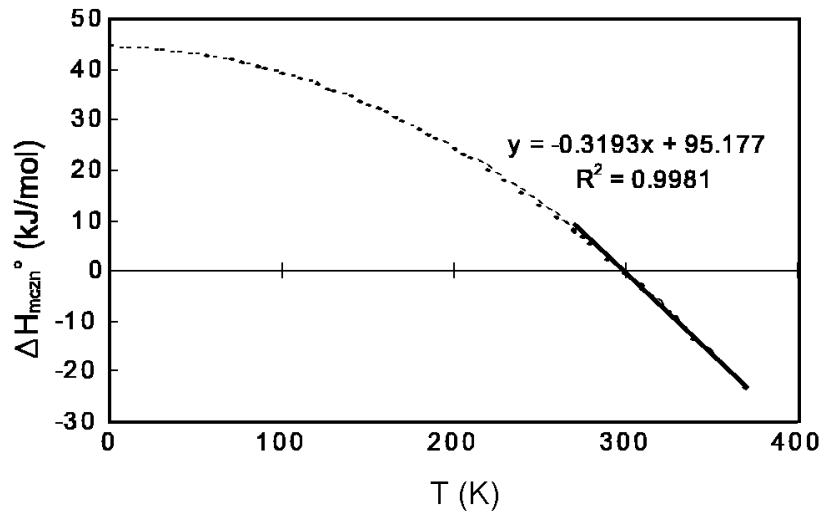

Figure 4. Micellization enthalpy $\Delta H_{m c z n}$ and its portion at $\mathrm{T}>273 \mathrm{~K}$.

$$
A \equiv \frac{a_{C p}-\text { const }_{S}}{R}, B \equiv-\frac{b_{C_{p}}}{2 R} . C \equiv-\frac{a_{C_{p}}}{R} . D \equiv \frac{\text { const }_{H}}{R}
$$

If we compare Eq. (32) to Eq. (36), then we can infer that the interaction parameter $\chi$ can be of the type

$$
\ddot{z}=A^{\prime}+B^{\prime} T+C^{\prime} \ln T+\frac{D^{\prime}}{T} .
$$

where the coefficient $A^{\prime}$ is a function of $A^{\prime}, \phi_{j}, 1^{\prime}$, and $B^{\prime}, C^{\prime}, D^{\prime}$ are functions of $\phi_{i}, B, \phi_{i} . C$, and $\phi_{i}, D$. respectively. Therefore. from $\mathrm{Eqs}$. (32) and (38) molar Gibbs free energy for a poly mer solution can be described as

$$
\begin{aligned}
\frac{\Delta \underline{G}_{m x}}{R T} & =\phi_{w} \ln \phi_{w}+\frac{\phi_{p}}{v^{\prime}} \ln \phi_{p} \\
& +\phi_{w} \phi_{z^{\prime}}\left(A^{\prime}+B^{\prime} T+C^{\prime} \ln T+\frac{D^{\prime}}{T}\right)
\end{aligned}
$$

Now we find that Eq. (26) is quite similar in form to Eq. (39). This implies that heat capacity change, $\Delta C_{\text {rimas. }}$ in micellization may be a linear function of temperature $\left(\Delta C_{P_{m}: \mathrm{s} / \mathrm{m}}=\right.$ $\left.a_{C p . m: n}+b_{C p . m: n} T\right)$. This in turn point to a quadratic dependence of the enthalpy change of micellization. $\underline{H}_{\text {minn }}$, on

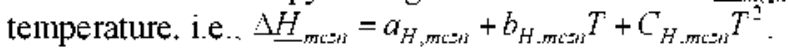

Experiments on micellization show that $\underline{H}_{m: n}$ is generally a linear function of temperature and that therefore $\Delta C_{P, m=3}$ is constant ${ }^{26-99}$ Hence, these observations appear to be inconsistent with the theoretical predictions. However, the real situation is like the one shown in Figure 4. which is prepared based on the CMC data of the cationic surfactant OTAC. When the temperature range extends to lower values below 0 ${ }^{\circ} \mathrm{C}(=273 \mathrm{~K}) . \underline{H}_{m c=n}$ shows clearly a second-order behavior. Micellizations are observed usually above $0{ }^{\circ} \mathrm{C}$ to avoid freezing of water. In this case the $\underline{H}_{m c s}$ curve above $0^{\circ} \mathrm{C}$ is almost like a line and it is. in fact. approximated by a line with the correlation coefficient of 0.9990 . This finding may support that the overall behavior of $\Delta C_{P \text { mizis }}$ for the micellization is linear with respect to temperature

\section{Conclusions}

The temperature dependence of CMC in micellization can be adequately described by Eq. (6). which has been derived statistical-mechanically. The prominent feature of Eq. (6) is that it yields the unique value of 2 for the power-law exponent n. without respect to surfactant species.

From the lattice-theoretic point of view. Eq. (6) may be based on the linear behavior of the heat capacity change of

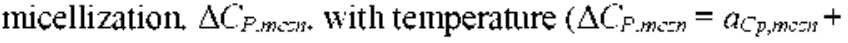
$\left.b_{(p, m i z} T\right)$. This points to a quadratic behavior of the enthalpy change of micellization. $\Delta \underline{H}_{m<s}$. Experimental measurements show that $\underline{H}_{m c=n}$ depends linearly on tenperature. It is. because the measurement temperatures are usually above $0^{\circ} \mathrm{C}(=273$ $\mathrm{K}$ ) of the freezing point of water. At these temperatures theoretical $\underline{H}_{m e n}$ also displays a linear behavior, which is evidenced by Figure 4 . Hence, all these support that the linear behavior of $\Delta C_{F}$ men may be responsible for the CMC of the type of Eq. (6).

\section{References}

1. Miller, D. D.; Magid, L. J. Evans, D. F. J. Phis. Chem. 1990 9-, 5921 .

2. Becher, P. Nonionic Surfactants; Schick, M. I., Ed.: Marcel Dekker: New York, 1967: Chapter 15.

3. Flockhart, B. D. J. Colloid Interface Sci. 1961, 16, 484

4. Stead, J. A.; Taylor, H. J. J. Colloil hnterface Sci. 1969, $30,482$.

5. Kim, H.-U.; Lim, K.-H. Colloid Siwf. 4 2004, 235, 121.

6. Kang, K.-H.: Kim, H.-U.: Lim, K.-H. Colloid Surf . 2001, 189, 113

7. Lim, K.-H. Manuscript in Preparation, 2009a.

8. Muller, N. Langmuir 1993, $9,96$.

9. Kim, H.-U.: Lim, K.-H. Bull Korean Chem. Soc. 2003, 2-, 1449.

10. Lim, K.-H.; Kang, K.-H.: Lee, M. J. J. Kor Ind Eng. Chem. 2006, 17, 625 .

11. Kang, K.-H.: Lim, K.-H. Kor Chem. Eng. Res. 2008, $46,824$.

12. Lim, K.-H. Manuscript in Preparation, 2009b.

13. Lim, K.-H. Statistical Themodynamics; Hantee Media, Seoul, Korea, 2008.

14. Kim, H.-U. Ph. D. Dissertation, Chung-Ang University, Seoul, Korea, 2002

15. La Mesa, C. J. Phys. Chem. 1990, 94, 323.

16. Stasiuk, E. N. B.; Schramm, L. L. J. Colloid Interface Sci. 1996, $324,178$.

17. Armarego, W. L. F.: Perrin D. D. Pmification of Laboratony Chemicals, $4^{\text {th }}$ ed.; Butternorth-Heinemann: Oxford, 1996.

18. La Mesa, C.: Ranieri, Z. A.; Terenzi, M. J. Sinface Sci. Tech. $1990,6,151$.

19. Shaw, D. J. Introduction to Colloid and Surface Chemistry, $4^{\text {th }}$ ed: Butternorth: 1991: p 91.

20. Evans, D. F.; Wennerstrom, H. The Colloidal Domain, where Phusics, Chemistry, Biology, and Techologn Heet; VCH: 1994.

21. Moro, Y. Aicelles, Theoretical and Applied Aspects; Plenum, 1992.

22. Tanford, C. Hydrophobic Effects; John Wiley and Sons. Inc: 1980.

23. Poland, D. C.; Schraga, H. A. J. Phis. Chem. 1965, 69, 2431

24. Nemethy, G.; Scluraga, H. A. J. Phis. Chem. 1962, 66, 1773.

25. Koningsveld, R. Br: Polym. J. 1975, 7, 435.

26. Koningsveld, R. Adw. Colloid Interface Sci. 1968, 2, 151

27. Kresheck, G. C.. Hargraves, W. A. J. Colloid Interface Sci. 1974, 48,481 .

28. Tomasic, V.: Chittofrati, A.; Kallay, N. Colloid Sinf. A 1995 , 104,95 .

29. Chen L-J.: Sheu Y.-H.: Li, P.-T. J. Phs Chem, 2004, $108,19096$. 